\title{
OSTEOGENESIS IMPERFECTA - CLINICAL AND MOLECULAR DIVERSITY
}

\author{
P.J. Roughley*, F. Rauch and F.H. Glorieux
}

Genetics Unit, Shriners Hospital for Children, Montreal, Canada

\begin{abstract}
Osteogenesis imperfecta is a heritable disorder of bone formation resulting in low bone mass and a propensity to fracture. It exhibits a broad range of clinical severity, ranging from multiple fracturing in utero and perinatal death to normal adult stature and a low fracture incidence. The disorder is currently classified into seven types based on differences in clinical presentation and bone architecture. Mutation in one of the type I collagen genes is commonly associated with osteogenesis imperfecta, but is not a prerequisite for the diagnosis. Indeed, the newer forms of osteogenesis imperfecta (types V, VI and VII) are not associated with type I collagen gene defects. Amongst the type I collagen gene mutations that can occur, missense base substitutions involving glycine codons in the exons encoding the central triple-helix forming domain predominate. Such mutations can occur in all the classical forms of osteogenesis imperfecta (types I-IV), but genotype/phenotype correlations are complex and often unpredictable. Treatment of osteogenesis imperfecta by bisphosphonate therapy can improve bone mass in all types of the disorder, and while not being a cure for the disorder does improve the quality of life of the patient.
\end{abstract}

Key Words: Osteogenesis imperfecta, bone, type I collagen, gene mutation, bisphosphonate therapy, classification.

*Address for correspondence:

P.J. Roughley

Genetics Unit, Shriners Hospital for Children

1529 Cedar Avenue, Montreal, Quebec H3G 1A6, Canada.

FAX Number: 514-842-5581

E-mail: proughley@shriners.mcgill.ca

\section{Introduction}

Osteogenesis imperfecta (OI) is a heritable disorder of bone formation that may affect more than 1:10,000 individuals. It is characterised by bone fragility due to low bone mass giving an increased fracture incidence (Kocher and Shaprio, 1998; Primorac et al., 2001). The bone fragility has led to the adoption of the trivial name of "brittle bone disease". The heritable nature of the disorder distinguishes it from idiopathic juvenile osteoporosis, although clinical osteoporosis is also a consequence of OI. Patients with OI do not have perturbations in serum calcium and vitamin D metabolite levels as a consequence of their disease, which distinguishes OI from osteomalacia.

Patients with OI may present with other clinical features, but these are by no means universal. Such features include the presence of blue sclera, dentinogenesis imperfecta, skin hyperlaxity and joint hypermobility. These are all features that one might expect to be associated with a disorder involving type I collagen, though such a relationship is not absolute. While individuals with OI having blue sclera and dentinogenesis imperfecta commonly have mutations in one of their type I collagen genes, there are many patients with type I collagen gene mutations who do not exhibit these secondary characteristics. In addition, the OI patient may present with Wormian bones in the sutures of the skull, and may be of decreased height and have skeletal deformity.

OI has been recognised as a disease entity since the 17 th century, when it was termed congenital osteomalacia. The term osteogenesis imperfecta was adopted in the late 19th century, and by the start of the 20th century the disorder had been subclassified into congenita and tarda forms to reflect the severe or mild disease that may be present in the young juvenile. As more patients with OI were investigated, the broad clinical spectrum of the disease was more fully recognised and more comprehensive classification systems were adopted. The system in common use today was presented by Sillence et al. (1979) and subdivides patients into four types based on disease severity and progression. However, it is appreciated that in reality the disorder represents a continuum of severity and that patients do not always fall conveniently into one clinical category. The application of modern bone histology techniques has also revealed that individuals with similar clinical presentation may exhibit vastly different changes in their bone architecture. This has led to further subdivisions of the disorder, with seven subtypes now having been defined.

One feature that is apparent from the preceding para- 
graph is that OI has never been defined using gene mutation as a criterion. Yet there has been a tendency in the past decade to try to redefine OI as a type I collagenopathy (Byers and Steiner, 1992; Prockop et al., 1993). This would imply that most if not all OI must arise from a mutation in either of the two genes for type I collagen. This is not the case, and while the majority of OI may arise from mutations in these genes there are a considerable number of individuals in whom such mutations are absent and mutation in a different gene is the causative event (Aitchison et al., 1988; Labuda et al., 2002; Wallis et al., 1993). A priori there is no reason why mutation in different genes cannot give rise to the same clinical phenotype, and therefore the definition of OI should remain a clinical one. However, it is certainly possible that in the future genetic diversity may result in a further subdivision of the disease classification.

\section{Clinical Diversity}

The classification system of Sillence et al. (1979) divides OI into four severity-based types, with the type IV category representing the clinically most diverse group. Prior to the analysis of bone architecture, the newer forms of OI (types V-VII) would all have fallen into the type IV category based on clinical presentation alone (Table 1).

\section{Type I OI}

Type I OI is the mildest form of the disease, being nondeforming and resulting in patients attaining a normal height. The patients typically have blue sclera, but dentinogenesis imperfecta is less common. Fractures are not commonly observed at birth, but begin with ambulation and subsequent falls during juvenile development, then commonly decrease following puberty.

\section{Type II OI}

Type II OI is the most severe form of the disease resulting in death in the perinatal period, with patients rarely surviving for more than a few days. The individuals exhibit multiple intrauterine rib and long bone fractures, and show severe skeletal deformity. Bone histology reveals a marked decrease in both cortical bone thickness and trabecular bone.

\section{Type III OI}

Type III OI is the most severe form of the disease compatible with survival past the perinatal period. It is characterised by severe progressive skeletal deformity that may have begun by birth. Individuals may possess multiple fractures at birth and suffer frequent fractures thereafter because of the highly fragile nature of their bones. The incidence of fracture remains high even in adult life. Individuals have severely short stature and because of their deformity and bone fragility are frequently confined to a wheelchair for life. Dentinogenesis imperfecta is commonly present.
Table I. Classification of $\mathrm{Ol}$

\begin{tabular}{|c|l|}
\hline Type & \multicolumn{1}{|c|}{ Typical Clinical Features } \\
\hline I & Non-deforming, normal height \\
II & Perinatal lethal \\
III & Severe deforming, very short \\
IV & Moderately deforming, variable phenotype \\
V & Moderately deforming, mineralized interosseous \\
& membrane, hyperplastic callus \\
VI & Moderately deforming, osteoid accumulation, \\
& abnormal lamellation \\
VII & Moderately deforming, short humeri/femora \\
\hline
\end{tabular}

\section{Type IV OI}

Type IV OI is clinically the most diverse group in the Sillence classification and encompasses all those individuals who do not meet the criteria for types I-III OI. The phenotype can vary from severe to mild, with the more severely affected patients presenting with fractures at birth, suffering moderate skeletal deformity and attaining a relatively short stature. Because of the phenotypic variability in this group, one might predict it to be the most likely source for genetic heterogeneity.

\section{Type V OI}

Type V OI is moderately deforming, and patients exhibit moderate to severe bone fragility (Glorieux et al., 2000). Blue sclera and dentinogenesis imperfecta are not present. The patients are characterised by three distinctive features: the presence of hypertrophic callus formation at fracture sites, calcification of the interosseous membranes between the bones of the forearm, and the presence of a radio-opaque metaphyseal band immediately adjacent to the growth plates upon X-ray. Upon histological examination, the lamellar organisation of the bone has an irregular mesh-like appearance distinct from the normal lamellar organisation (Figure 1).

\section{Type VI OI}

Type VI OI patients also present with moderate to severe skeletal deformity (Glorieux et al., 2002) and do not present with blue sclera or dentinogenesis imperfecta. The distinctive features of this OI type are the fish scale-like appearance of the bone lamellae and the presence of excessive osteoid upon histological examination (Figure 1). Although the osteoid accumulation suggests a mineralisation defect reminiscent of osteomalacia, there is no abnormality in calcium, phosphate, parathyroid hormone or vitamin D metabolism, and growth plate mineralisation proceeds normally.

\section{Type VII OI}

Type VII OI patients also have moderate to severe skeletal deformity and bone fragility, and lack blue sclera and 

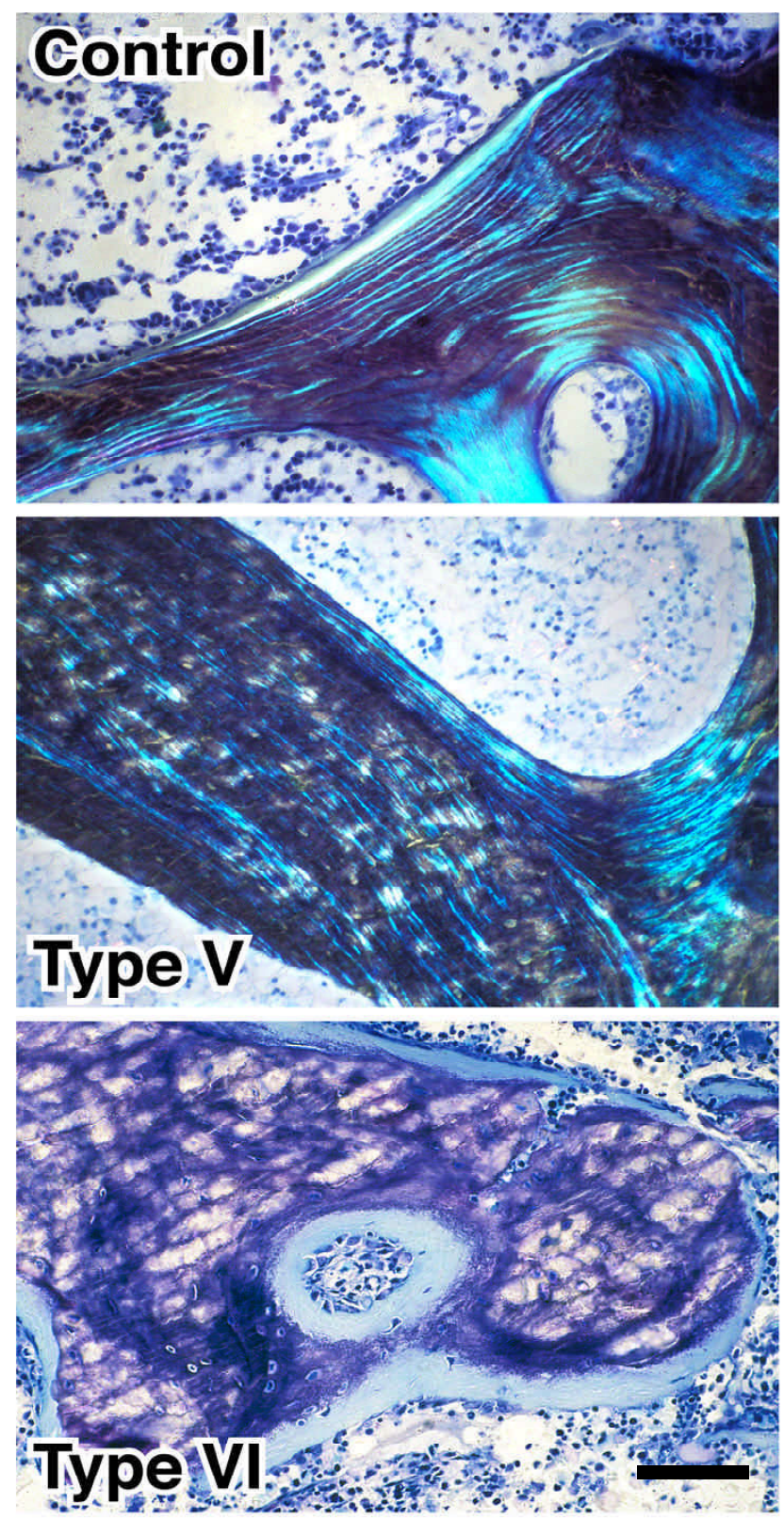

Figure 1: Lamellar appearance of trabecular bone. Iliac bone was fixed, stained (toluidine blue), and examined under polarized light. Scale bar $50 \mu \mathrm{m}$. dentinogenesis imperfecta. The distinctive clinical feature of the disease is a rhizomelic shortening of the humerus and femur (Ward et al., 2002). Unlike other forms of OI, which can be inherited in an autosomal dominant manner, type VII OI exhibits autosomal recessive inheritance.

\section{Genetic Diversity}

Types I-IV OI are commonly associated with mutations in the genes for type I collagen, whereas to date no type I collagen gene mutation has been detected in types $\mathrm{V}$ VII OI (Table 2). In the case of type VII OI, the existence of extended affected families has enabled the chromosome location of the gene defect to be mapped to chromosome 3p22-24.1 (Labuda et al., 2002). This locus is on a different chromosome from the type I collagen genes, and to date no plausible candidate gene has been identified in this region.

\section{Analysis of type I collagen gene mutations}

Owing to the large number of exons in the type I collagen genes, screening techniques have been employed to identify regions of the genes in which a mutation is present and so minimise the amount of nucleotide sequencing required (Nollau and Wagener, 1997; Prosser, 1993). A variety of different mutation screening techniques have been employed, including chemical cleavage of mismatches (CCM) (Bateman et al., 1993) and conformation sensitive gel electrophoresis (CSGE) (Ganguly et al., 1993; Korkko et al., 1998). The most widely used of these techniques is probably CSGE, because of its ease and speed of application and low cost. As with the other screening techniques, it does, however, suffer from the presence of false negative results. This is primarily due to the inability of a single electrophoresis condition to facilitate and maintain conformational changes in all heteroduplexes. While the use of multiple analysis conditions may overcome this problem, it is probably quicker to sequence all regions of the genes in those individuals who are

\section{Table 2. Gene Mutations Associated With OI}

\begin{tabular}{|c|c|c|}
\hline Type & COL1 Mutation & Characteristics \\
\hline I & Common & Often non-sense or frameshift in COL1A1 \\
II & Common & Often Gly missense in COL1A1 or COL1A2 \\
III & Common & Often Gly missense in COL1A1 or COL1A2 \\
IV & Common & Often Gly missense in COL1A1 or COL1A2 \\
V & Absent & \\
VI & Absent & \\
VII & Absent & Recessive, chromosome 3p22-24.1 \\
\hline
\end{tabular}


Table 3. Mutation Frequency In Type I Collagen Genes

\begin{tabular}{|c|c|c|}
\hline & COL1A1 & COL1A2 \\
\hline Total mutations detected & 54 & 55 \\
Gly mutations (helix) & 25 & 49 \\
Gly $\rightarrow$ Ser & 14 & 26 \\
Gly $\rightarrow$ Arg & 9 & 7 \\
Gly $\rightarrow$ Val & - & 6 \\
Gly $\rightarrow$ Asp & - & 3 \\
Gly $\rightarrow$ Glu & - & 3 \\
Gly $\rightarrow$ Cys & 1 & 2 \\
Gly $\rightarrow$ Ala & - & 1 \\
Gly $\rightarrow$ Trp & - & - \\
Gly $\rightarrow$ Pro & - & 1 \\
Non-sense mutations & 6 & - \\
Arg $\rightarrow$ Stop & 6 & - \\
Gln $\rightarrow$ Stop & - & - \\
Splicing mutation (ag...gt) & 9 & - \\
Frameshift deletion/insertion & 13 & 3 \\
In-frame deletion/insertion & 1 & \\
\hline
\end{tabular}

negative upon initial screening. Newer screening techniques, such as those employing denaturing high performance liquid chromatography (DHPLC), may be able to detect all mutations, but require a considerable financial outlay to purchase the requisite equipment.

CSGE therefore continues to be used for screening all OI patients for type I collagen gene mutations in this hospital. In individuals with types I, III and IV OI, mutations have been detected in one of the type I collagen genes in $50 \%, 66 \%$ and $80 \%$ of cases, respectively. Of the 36 out of 100 patients who were negative by CSGE screening, 9 have currently undergone full sequence analysis. In 4 of these cases, mutations not detected by CSGE were identified, but in the remaining 5 cases no type I collagen gene mutation could be identified. Thus, the deficiencies of the CSGE technique cannot be blamed for all the negative cases and it is likely that additional types of OI having a distinct genetic cause remain to be defined. To date 21 individuals with type V-VII OI have been analysed by CSGE, and all were negative for a type I collagen gene mutation. Of these, 5 individuals underwent full sequence analysis and all remained negative.

\section{Diversity of type I collagen gene mutations}

A multitude of different mutations have been described in the two type I collagen genes (COL1A1 and COL1A2) and are tabulated in the database of human collagen mutations (http://www.le.ac.uk/genetics/collagen) (Dalgleish, 1997). Mutations can involve both single nucleotide substitutions or base insertions and deletions, with missense mutations altering a glycine codon in the central exons of the genes encoding the protein region destined to form the characteristic collagen triple helix being most common. Replacement of either guanine $(\mathrm{G})$ residue in the glycine codon (GGN) can theoretically result in replace- ment by eight different amino acids (serine, cysteine, alanine, valine, aspartic acid, glutamic acid, arginine and tryptophan). All possibilities have been described and all result in an OI phenotype, though the frequency with which the different mutations occur varies considerably, with serine replacement being most common and tryptophan replacement being exceedingly rare.

Other mutations include nonsense substitutions in arginine or glutamine codons, missense mutation in the carboxy propeptides, frame-shift and in-frame insertions/ deletions in the coding sequence, and a variety of intron mutations that affect splicing. Two features of these mutations are of note for their absence. First, no COL1A1 or COL1A2 mutation affecting exons encoding the amino propeptides of type I collagen molecules (exons 1-6) have been associated with OI, and secondly, nonsense substitutions and frame-shift mutations have not been observed in the COL1A2 gene.

Analysis of 101 patients in this hospital having type I collagen mutations revealed that 74 had point mutations resulting in glycine substitutions (Table 3 ). This included 49 of 55 patients with a mutation in the COL1A2 gene. The remaining 6 individuals with COL1A2 mutations had either splicing mutations or in-frame deletions/insertions. For COL1A1 mutations, only 25 of 54 patients had a glycine substitution. The reason for this lower incidence is primarily due to the presence of 19 individuals with nonsense or frame-shift mutations. In all cases this latter group exhibited mild disease and in most cases they presented with a type I OI phenotype. The other mutation types were associated with types I, III or IV OI.

\section{Phenotype/genotype correlation}

Many investigators have attempted to correlate triple helical glycine mutation type and location with phenotypic 
severity (Byers and Steiner, 1992). This has proved to be an exceedingly difficult task, and there is no simple relationship that relates the type and position of a mutation in the helix to phenotype. Although some trends are apparent, such as phenotypic severity increasing with an amino to carboxy terminal orientation, with substitution by large and charged amino acids, and with COL1A1 versus COL1A2 mutations, there are many exceptions to these rules. There are a number of reasons to explain this variation. First, the collagen triple helix is not a uniform structure and exhibits regional differences in its stability and ability to accommodate mutations. This has led to the presentation of a regional model for OI severity, but full value of such a model awaits the identification of many more mutations to precisely define the different regions. Second, the intracellular consequences of a mutation are not constant, as different mutant messages or proteins can exhibit varying degrees of stability towards catabolism. This can influence the proportion of mutant collagen that is secreted by the osteoblast and also affect the synthesis of other gene products. Finally, gene mutations do not occur in isolation, but on a background of other factors that can influence bone strength. These factors may be due to genetic, nutritional or environmental variations between different individuals, and probably account for the fact that some commonly observed mutations can be associated with a variety of phenotypes.

\section{Medical Treatment}

The poor quality of the bone in OI patients is the major impediment to improving their quality of life, as it limits physical activity and impedes corrective orthopaedic surgery (Cinman, 2001; Engelbert et al., 1998; Zeitlin et al., 2003a). Thus a medical means of improving bone strength would be of considerable value. Several treatments have been tried to therapeutically augment bone with varying success (Byers, 2000). The greatest potential currently resides in bisphosphonate therapy (Fleisch, 1998). Once in blood, bisphosphonates avidly bind to the hydroxyapatite crystals of bone and act as anti-resorptive agents by disrupting osteoclast action. In so doing they can prevent the loss of both trabecular and cortical bone and result in an increased bone mass compared to that otherwise expected in the OI patient.

\section{Pamidronate therapy}

This hospital has been involved in a clinical trial using cyclic intravenous pamidronate administration to treat children with OI (Glorieux et al., 1998; Plotkin et al., 2000; Rauch et al., 2002, 2003a,b; Zeitlin et al., 2003b). Bisphosphonate treatment increased bone mineral density by increasing cortical bone width and the number of trabeculae present (Figure 2). As a consequence fracture incidence decreased. Perhaps more importantly to the more severely affected patients, the chronic bone pain that they experience subsided, permitting increased mobility and improvement in muscle strength. An increase in the rate of growth was also noted in those patients who were

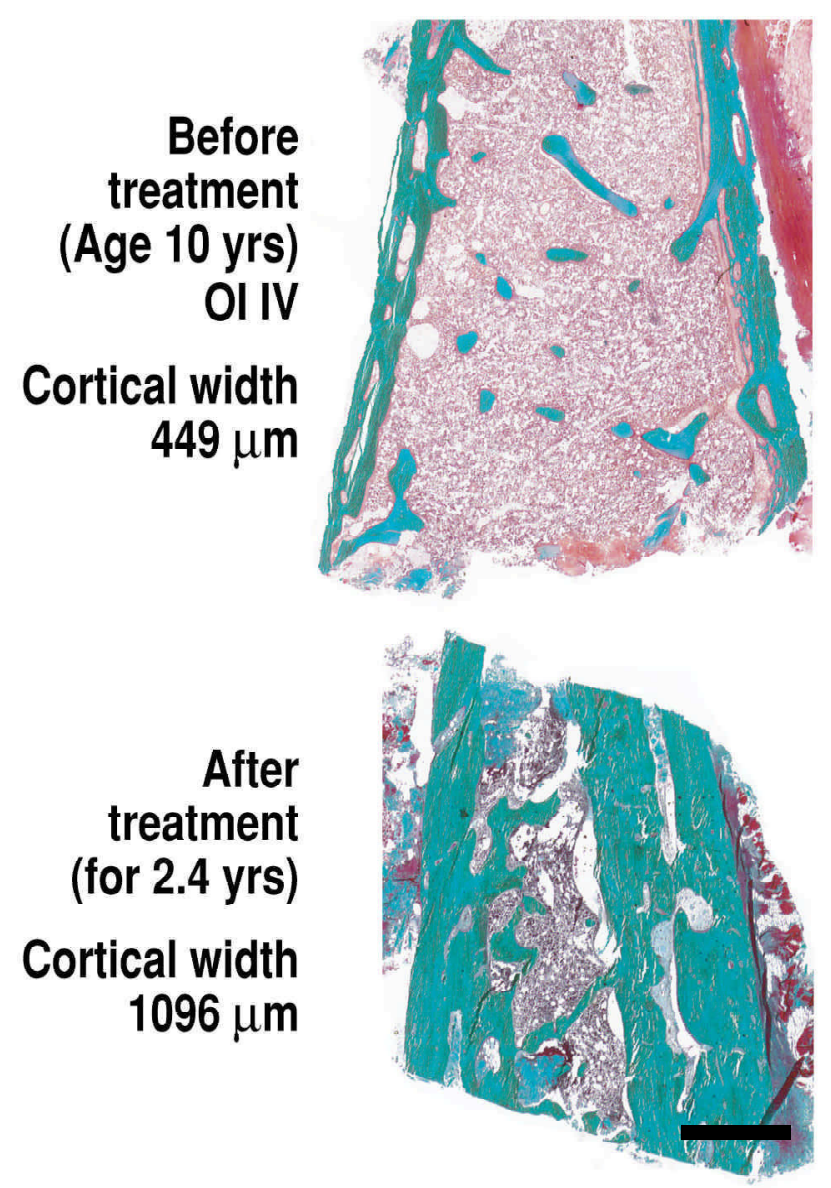

Figure 2: Effect of bisphosphonate on bone. Iliac bone was fixed, stained (Goldner-Masson trichrome), and examined by histomorphometry. Scale bar $1 \mathrm{~mm}$.

of diminished stature. In all patients, quality of life was increased as individuals were able to perform physical tasks previously unattainable. For example, wheelchair bound patients were now able to ambulate with the aid of a walker.

Bisphosphonate therapy in children cannot be viewed as a totally beneficial process (Burr, 2002; Parfitt et al., 1996; Srivastava and Alon, 1999). There are concerns with long term bisphosphonate use on the quality of bone that results when turnover is continuously suppressed, as such decreased remodelling may prevent repair of microdamage to the bone. As a consequence there may be limited benefit in continuing bisphosphonate therapy once growth has ceased and bone mineral density has plateaued. It is also possible that bisphosphonate therapy could impair the bone modelling process associated with fracture healing or surgical osteotomy. However, at present the benefits of bisphosphonate therapy by far outweigh its deficits.

\section{Future directions}

Bisphosphonate therapy is not a cure for OI, and while it improves bone strength by increasing the quantity of bone, it does not improve its quality as the gene defect is still present. It does, however, have the benefit of being applicable to all forms of OI, as it affects a common conse- 
quence of the disease not the gene mutation itself. Such disease-specific intervention will await the advent of effective and acceptable gene therapy (Forlino and Marini, 2000; Niyibizi et al., 2002).

\section{Conclusion}

OI is a heterogeneous disorder with a broad spectrum of clinical and genetic diversity. The clinical phenotype is a consequence of both the genetic diversity and non-genomic factors. The genetic diversity includes a multitude of different mutations in the genes for type I collagen, but is not confined to mutations in these genes. Irrespective of the genetic origin, the consequence is abnormal bone formation resulting in low bone mass and an increased risk of fracture. Identification and classification of OI is based on clinical and histological changes resulting from the perturbations in bone structure and are currently independent of the genetic origin. Current medical treatment with bisphosphonates aims to improve these perturbations in bone function and is independent of both phenotype and genotype.

\section{References}

Aitchison K, Ogilvie D, Honeyman M, Thompson E, Sykes B (1988) Homozygous osteogenesis imperfecta unlinked to collagen I genes. Hum Genet 78: 233-236.

Bateman JF, Lamande SR, Hannagan M, Moeller I, Dahl HHM, Cole WG (1993) Chemical cleavage method for the detection of RNA base changes: experience in the application to collagen mutations in osteogenesis imperfecta. Amer J Med Genet 45: 233-240.

Burr DB (2002) Targeted and nontargeted remodeling. Bone 30: 2-4.

Byers PH (2000) Osteogenesis imperfecta: perspectives and opportunities. Curr Opin Pediatr 12: 603-609.

Byers PH, Steiner RD (1992) Osteogenesis imperfecta. Annu Rev Med 43: 269-282.

Cinman N (2001) Osteogenesis imperfecta. A life not so fragile. Lancet 358 Suppl: S46.

Dalgleish R (1997) The human type I collagen mutation database. Nucleic Acids Res 25: 181-187.

Engelbert RH, Pruijs HE, Beemer FA, Helders PJ (1998) Osteogenesis imperfecta in childhood: treatment strategies. Arch Phys Med Rehabil 79: 1590-1594.

Fleisch H (1998) Bisphosphonates: mechanisms of action. Endocr Rev 19: 80-100.

Forlino A, Marini JC (2000) Osteogenesis imperfecta: prospects for molecular therapeutics. Mol Genet Metab 71: 225-232.

Ganguly A, Rock MJ, Prockop DJ (1993) Conformation-sensitive gel electrophoresis for rapid detection of single-base differences in double-stranded PCR products and DNA fragments: evidence for solvent-induced bends in DNA heteroduplexes. Proc Natl Acad Sci 90: 1032510329.

Glorieux FH, Bishop NJ, Plotkin H, Chabot G, Lanoue G, Travers R (1998) Cyclic administration of pamidronate in children with severe osteogenesis imperfecta. N Engl J
Med 339: 947-952.

Glorieux FH, Rauch F, Plotkin H, Ward L, Travers R, Roughley P, Lalic L, Glorieux DF, Fassier F, Bishop NJ (2000) Type V osteogenesis imperfecta: a new form of brittle bone disease. J Bone Miner Res 15: 1650-1658.

Glorieux FH, Ward LM, Rauch F, Lalic L, Roughley PJ, Travers R (2002) Osteogenesis imperfecta type VI: a form of brittle bone disease with a mineralization defect. J Bone Miner Res 17: 30-38.

Kocher MS, Shapiro F (1998) Osteogenesis imperfecta. J Am Acad Orthop Surg 6: 225-236.

Korkko J, Ala-Kokko L, De Paepe A, Nuytinck L, Earley J, Prockop DJ (1998) Analysis of the COL1A1 and COL1A2 genes by PCR amplification and scanning by conformation-sensitive gel electrophoresis identifies only COL1A1 mutations in 15 patients with osteogenesis imperfecta type I: identification of common sequences of null-allele mutations. Am J Hum Genet 62: 98-110.

Labuda M, Morissette J, Ward LM, Rauch F, Lalic L, Roughley PJ, Glorieux FH (2002) Osteogenesis imperfecta type VII maps to the short arm of chromosome 3. Bone 31: 19-25.

Niyibizi C, Wallach CJ, Mi Z, Robbins PD (2002) Approaches for skeletal gene therapy. Crit Rev Eukaryot Gene Expr 12: 163-173.

Nollau P, Wagener C (1997) Methods for detection of point mutations: performance and quality assessment. Clin Chem 43: 1114-1128.

Parfitt AM, Mundy GR, Roodman GD, Hughes DE, Boyce BF (1996) A new model for the regulation of bone resorption, with particular reference to the effects of bisphosphonates. J Bone Miner Res 11: 150-159.

Plotkin H, Rauch F, Bishop NJ, Montpetit K, RuckGibis J, Travers R, Glorieux FH (2000) Pamidronate treatment of severe osteogenesis imperfecta in children under 3 years of age. J Clin Endocrinol Metab 85: 1846-1850.

Primorac D, Rowe DW, Mottes M, Barisic I, Anticevic D, Mirandola S, Gomez Lira M, Kalajzic I, Kusec V, Glorieux FH (2001) Osteogenesis imperfecta at the beginning of bone and joint decade. Croat Med J 4: 393415.

Prockop DJ, Colige A, Helminen H, Khillan JS, Pereira R, Vandenberg P (1993) Mutations in type I procollagen that cause osteogenesis imperfecta: effects of the mutations on the assembly of collagen into fibrils, the basis of phenotypic variations, and potential antisense therapies. J Bone Miner Res 8 Suppl 2: S489-S492.

Prosser J (1993) Detecting single-base mutations. Trends Biotechnol 11: 238-246.

Rauch F, Travers R, Plotkin H, Glorieux FH (2002) The effects of intravenous pamidronate on the bone tissue of children and adolescents with osteogenesis imperfecta. J Clin Invest 110: 1293-1299.

Rauch F, Plotkin H, Travers R, Zeitlin L, Glorieux FH (2003a) Osteogenesis imperfecta types I, III and IV: effect of pamidronate therapy on bone and mineral metabolism. J Clin Endocrinol Metab 88: 986-992.

Rauch F, Plotkin H, Zeitlin L, Glorieux FH (2003b) Bone mass, size and density in children and adolescents with osteogenesis imperfecta: Effect of intravenous 
pamidronate therapy. J Bone Miner Res 18: 610-614.

Sillence DO, Senn A, Danks DM (1979) Genetic heterogeneity in osteogenesis imperfecta. J Med Genet 16: 101-116.

Srivastava T, Alon US (1999) Bisphosphonates: from grandparents to grandchildren. Clin Pediatr (Phila) 38: 687-702.

Wallis GA, Sykes B, Byers PH, Mathew CG, Viljoen D, Beighton P (1993) Osteogenesis imperfecta type III: mutations in the type I collagen structural genes, COL1A1 and COL1A2, are not necessarily responsible. J Med Genet 30: $492-496$.

Ward LM, Rauch F, Travers R, Chabot G, Azouz EM, Lalic L, Roughley PJ, Glorieux FH (2002) Osteogenesis imperfecta type VII: an autosomal recessive form of brittle bone disease. Bone 31: 12-18.

Zeitlin L, Fassier F, Glorieux FH (2003a) Modern approach to children with osteogenesis imperfecta. J Pediatr Orthop B 12: 77-87.

Zeitlin L, Rauch F, Plotkin H, Glorieux FH (2003b) Height and weight development during long-term therapy with cyclical intravenous pamidronate in children and adolescents with osteogenesis imperfecta types I, III and IV. Pediatrics 111: 1032-1036.

\section{Discussion with Reviewers}

W. Hofstetter: Are there any reasons for the observed impairment of mineralisation in type VI OI, and can this be treated with supplementation of $\mathrm{Ca}$ and vitamin $\mathrm{D}$ ?

Authors: The observed impairment of mineralisation in type VI OI may be due to a bone matrix defect. It should be noted that the mineralisation defect affects only bone matrix (resulting in osteomalacia), but not the growth plate (thus, there is no rickets). Serum levels of calcium and vitamin D are normal in these children when adequately nourished. Treatment with calcium and vitamin D above recommended daily allowance doses has not been tried.

W. Hofstetter: Might the authors speculate on the role of "silent" mutations, i.e. mutations that do not show a phenotype during development and adulthood, but that might play a role in ageing, i.e. osteoporosis and osteoarthritis? Authors: Mild cases of OI may remain undiagnosed during childhood, especially when the family history is nega- tive. The incidence of fractures in mild OI is low during young adulthood, but increases with ageing. It is thus possible that some patients who are classified as having postmenopausal osteoporosis actually suffer from mild OI. However, careful history and clinical examination should usually lead to the correct diagnosis.

W. Hofstetter: Are there other bisphosphonates than pamidronate used in the treatment of patients with OI? Success?

Authors: There are a number of case reports where OI patients have been treated with bisphosphonates other than pamidronate. Apart from that there is one Italian study on the use of intravenous neridronate in adults with OI (Adami et al., 2003). A lower fracture rate was reported during than before treatment.

M. Alini: Could the authors explain or speculate how bisphosphonates act to increase growth rate in treated children? Intuitively, the contrary should occur.

Authors: Children with severe OI who are treated with pamidronate have fewer fractures and more normally shaped vertebral bodies than untreated children. These factors may explain the observed positive height development during treatment.

M. Alini: Are there any differences between the different types of OI in response to bisphosphonate therapy?

Authors: It appears that patients with type VI OI respond less well to therapy than the other patients. We have not detected clear differences between the other types. It should be noted that scientific data are limited to children with moderate to severe OI. There is no information whether children with mild OI (with two or fewer fractures per year, no vertebral compression fractures, and no long bone deformities) have any benefit from bisphosphonate treatment.

\section{Additional Reference}

Adami S, Gatti D, Colapietro F, Fracassi E, Braga V, Rossini M, Tatò L (2003) Intravenous neridronate in adults with osteogenesis imperfecta. J Bone Miner Res 18: 126130 . 